\title{
PastryStrings: A Comprehensive Content-Based Publish/Subscribe DHT Network
}

\author{
Ioannis Aekaterinidis and Peter Triantafillou \\ RA Computer Technology Institute and \\ Dept. of Computer Engineering and Informatics, \\ University of Patras, Greece \\ \{aikater,peter\}@ceid.upatras.gr
}

\begin{abstract}
In this work we propose and develop a comprehensive infrastructure, coined PastryStrings, for supporting rich queries on both numerical (with range, and comparison predicates) and string attributes, (accommodating equality, prefix, suffix, and containment predicates) over DHT networks utilising prefix-based routing. As event-based, publish/subscribe information systems are a champion application class, we formulate our solution in terms of this environment.
\end{abstract}

\section{Introduction}

Peer-to-peer (p2p) data networks are appropriate for building large-scale distributed systems and applications since they are completely decentralised, scalable, and self organising. All participating nodes have equal opportunities and are providing services where information is exchanged directly between them. There are two families of $\mathrm{p} 2 \mathrm{p}$ networks: structured, where the data placement and the network topology are tightly controlled, and the unstructured ones. The most prominent structured $\mathrm{p} 2 \mathrm{p}$ networks are built using a Distributed Hash Table (DHT [1, 19-22,31]). A special class of DHTs employ prefix-based routing based on Plaxton's et al. Mesh [17] (Tapestry [31], Pastry [21], Bamboo [20]). DHT architectures provide scalable resource look-up and routing with $O(\log (N))$ complexity in a $N$ node network.

A large body of research is currently targeting the extension and employment of $\mathrm{p} 2 \mathrm{p}$ data network architectures over either unstructured or DHT-based p2p networks ([7, 11-13, 15, 18,30]). Related work has provided solutions for a large number of problems, from architectures and algorithms for searching relevant data, to range query processing and data integration, and has started to examine how to support join and aggregate queries. This fact testifies to the importance the distributed systems community at large is giving to being able to support data-intensive applications over large-scale network infrastructures.

Supporting a 'rich' set of queries (queries involving prefix, suffix, containment, and equality predicates on strings, and range and comparison predicates on numerical-typed attributes) in $\mathrm{p} 2 \mathrm{p}$ data networks may be very useful to a number of applications. A representative class of such distributed applications is systems built using the publish/subscribe (pub/sub) paradigm. With our work in this paper we contribute a comprehensive infrastructure, coined PastryStrings, supporting efficiently and scalably a rich set of operators on string and numerical-typed attributes. Given the popularity of the pub/sub paradigm, we focus on it and formulate our solution in terms of this environment.

\section{Background and contributions}

\subsection{Plaxton's mesh and Pastry}

Pastry [21], as well as Tapestry [31] and Bamboo [20], are all based on location and routing mechanisms introduced in [17].

Plaxton et. al. present in [17] a distributed data structure (a.k.a. Plaxton Mesh) optimised for routing and locating objects in a very large network with constant size routing tables. Assuming a static network, routing tables consist of multiple levels, where in each level $i$ there are pointers to nodes whose identifiers (or node ids) have the same $i$-digit long suffix with the current node's id. The routing of messages is achieved by resolving one digit of the destination id in each step $i$ and looking at the $i+1$ level of the local routing table for the next node. This mechanism ensures that a node will be reached in at most $m=\log _{\beta}(N)$ logical hops, where $N$ is the namespace size, $\beta$ is the base of ids, and $m$ the number of digits in an id. The size of the routing table 
is constant and equal to $\beta \times \log _{\beta}(N)$.

Pastry [21] offers a robust, scalable, and self-organising extension to Plaxton's Mesh under a dynamic environment. The routing scheme in Pastry, is similar to the one proposed by Plaxton et. al. with routing tables of size $\beta \times \log _{\beta}(N)$ (with $\log _{\beta}(N)$ levels/rows and $\beta$ columns per level), resulting in $\log _{\beta}(N)$ logical hops to locate a node. However, prefix (instead of suffix) matching is performed in each routing step towards the destination node, while routing table entries point to the closest node with the appropriate id prefix in terms of a proximity metric (such as round-trip time, RTT). Moreover, in order to achieve reliable routing, there is the notion of a leaf set for each node consisting of $L$ pointers to nodes with id numerically close to the current node's id. In Pastry there is also the notion of neighbouring nodes, which is a set of $M$ pointers to nearby nodes according to a proximity metric and used for maintaining locality properties. Tapestry [31] and Bamboo [20] are DHTs with similar routing functionality.

\subsection{The publish/subscribe paradigm}

In the pub/sub paradigm, subscribing users are interested in particular events, comprising a small subset of the set of all events that publishing users may generate. Pub/sub systems [9] are separated in two major categories, according to the way subscribers express their interests; the topic-based and the content-based pub/sub systems. Content-based pub/sub systems are preferable as they give users the ability to express their interest by issuing continuous queries, termed subscriptions, specifying predicates over the values of a number of well defined attributes. The matching of publications (a.k.a. events) to subscriptions (a.k.a. interests) is done based on the content (values of attributes).

The main challenge in a distributed pub/sub environment is the development of an efficient distributed matching algorithm and related efficient algorithms to store subscriptions in the network. Distributed solutions have been provided for topic-based pub/sub systems [6]. More recently, some attempts on distributed content-based pub/sub systems use routing trees to disseminate the events to interested users based on multicast techniques $[4,5,8,13,23,24,26]$. Typically, processing subscriptions and/or events in these approaches requires $O(N)$ messages in $N$-node networks. Additionally, there exist techniques for subscription summarization that significantly reduce the complexity $[26,27]$.

Some other attempts use the notion of rendezvous nodes which ensure that events and subscriptions meet in the system [16]. Some approaches have also considered the coupling of topic-based and content-based systems [32] where events/subscriptions are automatically classified in topics. However, none of these works supports string attributes with prefix, suffix, and containment predicates.
Finally, some techniques found in the literature for string indexing may also be relevant to our goals. The most promising is the technique relying on n-grams [12] which can be applied for substring matching. However, deciding on the right value of $n$ of $n-$ grams is difficult. Thus, typically, several values of $n$ are used, which has a multiplicative effect on the overheads associated with $n$-grams. A relevant to PastryStrings work is presented in [28] where $\mathrm{pub} / \mathrm{sub}$ functionality is offered on top of the Chord DHT using an attribute-value model, called AWPS. In [14] a balanced tree structure on top of a p2p network is presented, which can handle equality and range queries.

Prior research aiming to address relevant issues of processing 'rich' queries as typified by those in a pub/sub environment and which is closest to this work includes our previous work to support numerical-attributes in a DHT-based pub/sub environment [25]. In this work we showed how to exploit DHTs and order-preserving hashing to process range subscriptions (with range $R$ of size $|R|$ ), with worstcase message complexity $O(|R|+\log (N))$ and events in $O(\log (N))$, in an $\mathrm{N}$-node network. Our more recent work in [2] presented an approach able to support string-attribute predicates with message complexity $O(l \times \log (N))$ (where $l$ is the average length of string values) for events and $O(\log (N))$ for subscriptions. Both of these works were DHT-independent, relying on the underlying DHT's lookup functionality for routing events and subscriptions. In the same spirit, the work in [18] proposed a distributed trie structure called Prefix Hash Tree (PHT) which is built on top of a DHT p2p network and can support range queries and prefix string queries. PHT, like [2] and [25] enjoy universal applicability (as they are based solely on the DHT's lookup function). However, it too suffers from a number of drawbacks regarding its performance and particularly the message complexity of processing range and string queries. Adapting PHT to the pub/sub paradigm we would observe that range query (subscription) processing would require $O(\log (N)+|R| \times \log (N))$ messages. This is similar to the performance of [25], only because of the use of orderpreserving hashing the latter work has $O(|R|+\log (N))$ complexity (since peer nodes storing neighbouring values are network neighbours due to the order-preserving data placement). With respect to processing events matching prefix-string subscriptions (in general) PHT would exhibit a message complexity of $O(l \times \log (N))$, similar to [2], since one DHT lookup is needed per character of the string. The reader should note that the value of $|R|$ can be large and that $l$ is typically in the order of $\log (N)$.

\subsection{Contribution}

What is very much lacking in the literature is a single unified, comprehensive DHT-based, pub/sub architec- 
ture that can support with the same structures both string and numerical-attribute events and subscriptions effectively. This implies that it is highly desirable to offer logarithmic event and subscription processing performance for both string and numerical attributes.

\section{PastryStrings architecture and rationale}

The two primary design choices that best describe PastryStrings are (i) a tuned Pastry (or any other Plaxton-like DHT) network with an alphabet base ${ }^{1} \beta$ appropriately defined so as to map string values (every possible spoken word) to nodes and (ii) a tree structure (known as string trees) on top of Pastry dedicated for storing subscriptions and matching events to subscriptions using prefix-based routing a la Pastry.

Each tree in the string tree forest is dedicated to one of the $\beta$ characters of our alphabet. For string queries starting with a specific character we will first locate the appropriate tree dedicated to that character and follow a path towards the "rendezvous node" inside that tree where events and subscriptions will meet. Each node in a string tree uses the Pastry nodes' local routing tables as a hint for the tree construction.

The architecture of PastryStrings consists of clients that are producers/consumers, issuing events/subscriptions, respectively. Each client is "attached" to a Pastry network node using any appropriate mechanism. Each Pastry node hosts one or more string tree nodes responsible for holding and processing events and subscriptions.

Consumers publish their interests with subscriptions that are stored in specific string tree nodes, the "rendezvous nodes". Producers generate events that are delivered only to interested consumers by collecting and 'activating' the already stored subscriptions in the "rendezvous nodes". For simplicity of presentation, we will concentrate in this section on a single-attribute event/subscription schema.

\subsection{String trees}

There are two types of nodes in PastryStrings. Network (Pastry) nodes (referred to as simply "nodes") and string tree nodes (referred to as Tnodes). A node in general hosts several Tnodes. Nodes have ids (assigned by Pastry) while Tnodes have labels for identifying them. A Tnode's label is in general a prefix-string that is meant to identify a specific Tnode that is responsible for storing subscriptions matching the string label. In this case, the Tnode with label 'lbl' is denoted as Tnode $l b l$. Each string tree is denoted by $T_{i}$ where $i \in S=$

\footnotetext{
${ }^{1}$ The digit base is equal to 64 as a result of the $2 \times 26=52$ characters of the English alphabet (uppercase and lower case), the 10 numerical characters and two special symbols: space and period.
}

$\{a \mid a$ is one of the $\beta$ characters of the alphabet $\}$ is the character for which $T_{i}$ is responsible for. This means that every label in the $T_{i}$ tree starts with the same character $i$. Each $T_{i}$ has a maximum depth (root's depth is zero) equal to the maximum allowable string-length.

A first attempt regarding the string tree construction is to take advantage of the routing table of each node in the Pastry network (e.g. with id digit base $\beta=64)^{2}$ and use those routing tables for (prefix-based) routing the queries to rendezvous Tnodes. However, this turns out not to be a good idea due to the complications introduced by the maintenance functionality in the presence of churn. Consider, for example, the case where a Pastry node $A$ changes the entry in its routing table which was pointing to $B$, to point now to $C$ because of $B^{\prime} s$ departure. Then, the entire subtree rooted at $B$ would become unreachable. In this case, either this subtree should be moved to hang from $C$, (which implies that every Tnode in the subtree would have to be replaced and be hosted by a Pastry node reachable from $C$ ) or $C^{\prime} s$ routing table should be updated, which implies that we would interfere with the way the Pastry network is constructed.

Thus, a better idea is to maintain an additional routing table for each Tnode of our $T_{i}$ trees of constant length equal to $\beta$ with entries pointing to the Tnode's children. The construction of this routing table for a Tnode is done based on the routing table of the Pastry node hosting the Tnode. The routing table at each Pastry node holds $\beta \times \log _{\beta}(N)$, entries. Each Tnode uses as a hint one of the $\log _{\beta}(N)$ levels in the routing table of the Pastry node, where the Tnode is hosted, in order to build its own routing table. More precisely, if a Tnode lays in depth $d$ then it is going to use the $(d+1) \bmod \log _{\beta}(N)$ level of the host's routing table. Since the alphabet for Pastry node ids and Tnode's labels is the same, the aim is to do prefix-based routing over Tnode's labels utilising the Pastry infrastructure for doing prefix-based routing over node ids, so as (i) to leverage the Pastry selforganisation logic and (ii) achieve short RTT where possible.

String trees are created dynamically as new requests for storing subscriptions with string-valued predicates, arrive. Since there are many string trees we locate the root of a specific $T_{i}$ by hashing the first character of the given string with a uniformly-distributed hash function like SHA1. Then, the node with that id will host (or already hosts) the root of $T_{i}$. The following example illustrates how string trees are created.

\section{Example 1 Simple subscription storing. In Figure 1 a}

${ }^{2}$ If, for instance $\beta=64$ and str Length, the maximum string length, is 20 , then the namespace size is $64^{20} \cong 2^{120}$. If $\beta=64$ and str Length $=30$ then the namespace size is $64^{30} \cong 2^{180}$. Please note, that typical DHTs are reported to have a namespace size in the range of $2^{128} \ldots 2^{164}$ 
simplified snapshot of the PastryStrings infrastructure is presented. In this example, we use 3-character long ids with alphabet of base $\beta=2$. Suppose now that a user expresses her interests with two subscriptions (with identifiers $S u b I D_{1}$ and $S u b I D_{2}$ ) on the same attribute, setting the attribute's value to '010' and '00' respectively.

In general, the string tree forest consists of two trees: the $T_{0}$ and $T_{1}$. Both subscriptions in this example concern $T_{0}$. To process the storage request for value '010' we should first locate the Pastry node responsible for hosting the root of the $T_{0}$ tree (say node with id '000) by hashing the character ' $O$ ' and locating the Pastry node with id equal (or close) to the hashing result. We construct there the root, Tnode ${ }_{0}$ and since its routing table is empty we use the host's routing table at level 1 as a hint (recall that the root lays in depth $d=0$ and thus we look at level $(d+1) \bmod 3=1)$. The routing table of the host of Tnode $_{0}$ at level 1 has two entries (since we have a binary alphabet) which are copied to Tnode, 's routing table. The first one is pointing to node '001' which will host Tnode 00 , while the second entry points to '011' which is going to host Tnode $_{01}$. Now that we have filled Tnode 0 's routing table we further process the request by checking the second character of the string, '1'. We look at the root's routing table, at column ' 1 ', and select the record that matches the next digit, sending the request to the node '011'. Since initially node '011' does not host any Tnode, we construct there Tnode $_{01}$ and fill its routing table with the level 2 of its host's routing table. Then we process the storage request, by examining the final character of the string, ' $O$ '. We again ask Tnode ${ }_{01}$ for its pointer in column ' 0 ' of its routing table and we forward the request to node '100'. We construct there Tnode 010 and store SubI $D_{1}$. The second storage request is handled similarly, as you can see in Figure $1 . \diamond$

\subsection{Event and subscription rendezvous}

When an event arrives defining a value in our simple single-attribute event/subscription schema, we locate the root of the appropriate $T_{i}$ and forward the event towards the Tnode that has the same label as the string value in the event, by resolving one character at a time. All subscriptions found there, are considered to match the event since they have declared the same value as the event.

Example 2 Simple event-subscription rendezvous. Figure 2 shows a snapshot of PastryStrings with two subscriptions already stored from the previous example. Suppose that an event defining the value '010' arrives at the system. We first locate the root node, Tnode $e_{0}$, of the string tree responsible for the character ' $O$ ' and send there the event. Tnode 0 will look-up its routing table in column ' 1 ' for the pointer to the nearby Tnode 01 and will send there the event. Tnode 01 will look-up its own routing table in column ' $O$ ', for the Tnode 010 where the subscription SubID $D_{1}$ is stored.

Having introduced the notion of string trees, $T_{i}$, and how event routing is performed, we see that two different routing schemes coexist in PastryStrings. Specifically:

- Pastry Routing: is done based on Pastry's routing tables and offers the common API functions described in [21]. Pastry Routing is necessary for locating the $T_{i}$ trees, and for creating string tree paths.

- String Tree Routing: is performed within a $T_{i}$ tree and exploits the Tnodes' routing tables in order to forward the requests towards the leaves. A typical API function is $T c_{-}$forward (msg, key), performed locally at each Tnode, forwarding a message $(\mathrm{msg})$ to the Tnode that is responsible for the value key.

We stress that Pastry routing is unaffected by PastryStrings. String tree routing uses the Tnodes's routing tables. A Tnode's routing table in essence constitutes another routing level, having one entry for each possible string character value.

\subsection{Supporting complex string and nu- merical predicates}

In this section we will show how to support prefix (e.g. 'abc*') and suffix (e.g. '*abc') predicates on string attributes as well as range (and comparison $\leq, \geq, \neq$ ) queries on numerical attributes over the PastryStrings infrastructure.

\subsubsection{Storing subscriptions and processing incoming events: string-typed attributes}

First note that a suffix operation can be easily transformed into a prefix operation if we simply proceed to examine the string from its last to its first character. Thus, without loss of generality, we shall only present how a prefix operation on string values can be applied in PastryStrings.

Suppose that we have a subscription with a prefix predicate. In order to appropriately store the subscription we follow the same methodology as if we had an equality predicate. Now when an event arrives, we locate the appropriate $T_{i}$ tree which is responsible for the first character of the event's string value (e.g. $T_{a}$ for predicate $a b c^{*}$ ), and we then traverse a specific path of the tree (from the root Tnode towards the leaves) until we find the Tnode whose label is that value. During this traversal and since we perform prefix-based routing, all Tnodes belonging to this path may be storing subscriptions matching a prefix of the event's value. 


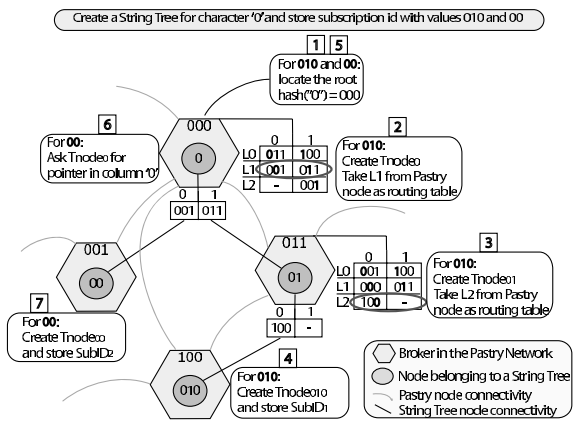

Figure 1. String tree construction and subscription storing.

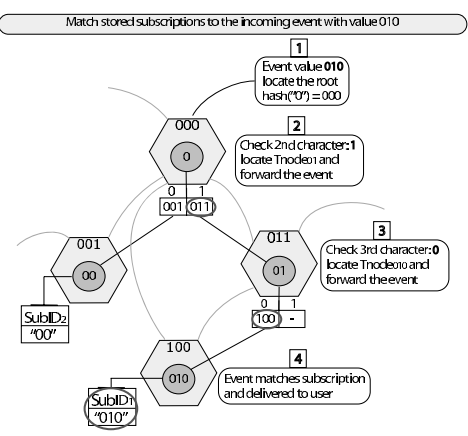

Figure 2. String-typed event to subscription matching.

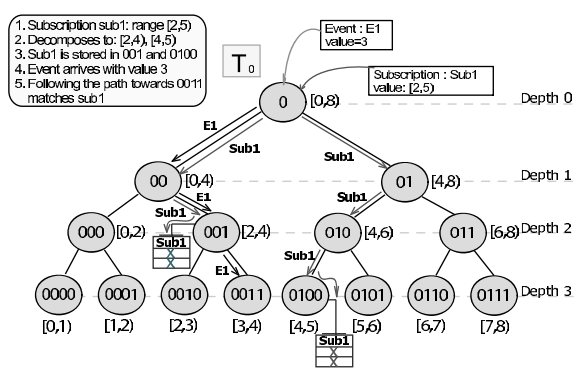

Figure 3. Numerical-typed event to subscription matching.
In addition to prefix and suffix predicates, our scheme can also support a "containment" predicate (e.g. ' $a * c$ '). This containment operator can be easily decomposed to prefix/suffix operations. The main idea is that, for example, ' $a * c$ ' can be viewed both as prefix (i.e. ' $a$ *') and suffix (i.e. ' $* c^{\prime}$ ') predicates and with appropriate post-processing we can conclude on possible matching. Due to space limitations we omit the detailed methodology for this, which is straightforward extension given the support for prefix/suffix predicates.

\subsubsection{Storing subscriptions and processing incoming events: numerical-typed attributes}

PastryStrings also supports numerical attributes with range, $\leq, \geq, \neq$, and $=$ predicates. The key idea here is that every possible range of integer values may be appropriately mapped to a number of Tnodes based on their labels and their location in the $T_{i}$ tree. There are several ways to do so. Here we adapt the Range Search Tree (RST) approach presented in [10], and encapsulate its functionality within PastryStrings.

The required functionality consists of: (i) assign subranges of numerical values to Tnodes and (ii) partitioning a given range into appropriate subranges. Given this functionality, when a subscription arrives declaring a range of values, we first decompose the range to proper sub-ranges. Then we locate the appropriate for each subrange Tnode in $T_{i}$ and store there the SubID. When an event arrives declaring a specific value, we transform the value to $\beta$-ary string representation, locate the appropriate $T_{i}$ tree and follow the path from the root to the leaf with the same label as the given $\beta$-ary string. Each Tnode in this path may have stored SubIDs declaring ranges including the value of the event.

Recall that each $T_{i}$ tree has a depth $D$ ( $D$ equals the maximum string length). $d$ denotes the depth of Tnode $l b l$. We also denote with num $(l b l)$ the numerical representation of the label string $l b l$. For instance, given $\beta=2$ and $l b l=$ 0010 then $\operatorname{num}\left({ }^{\circ} 0010^{\prime}\right)=2$.

With the proposed scheme, domains of size up to $\beta^{D}$ may be easily mapped to Tnodes in the PastryStrings infrastructure. Each Tnode ${ }_{l b l}$ (in depth $d$ in a $T_{i}$ tree) responsible for the prefix $l b l$ is assigned by RST to hold a specific range of values belonging to the following set:

$$
\left[n u m(l b l) \times \beta^{D-d-1},(\operatorname{num}(l b l)+1) \times \beta^{D-d-1}\right)
$$

In Figure 3 we present a simplified version of the PastryStrings infrastructure for explaining the main functionality. In this example $\beta=2$ and string length equal to $4(D=4)$. As you can see, Tnode $_{001}$, is going to store ranges belonging to the interval $[2,4)$. Using the node to interval mapping above, we get: num( "001") $=1, \beta=2, D=4$ and $d=2$ resulting in: $\left[1 \times 2^{4-2-1},(1+1) \times 2^{4-2-1}\right)=[2,4)$. Each leaf Tnode holds the smallest possible sub-range while any non-leaf Tnode holds the union of its children subranges. The union of sub-ranges of all root Tnodes of our schema, covers the maximum possible domain for integer values (with size $\beta^{D}$ ).

Up to now we set our $T_{i}$ trees so as to easily discover the sub-range a Tnode is responsible for, based on the Tnode $_{l b l}$ label. For storing a subscription with a range predicate, we should actually break the range into subranges, find the appropriate Tnodes responsible for each subrange, and then store there the $S u b I D$ of the subscription with the range predicate. In [10], a specific algorithm for this purpose is developed with $O\left(\log _{\beta}(|R|)\right)$ complexity for a range $R$ with length $|R|$. Using this algorithm (with small modifications) we can decompose for example the range $[2,5)$ into $[2,4)$ and $[4,5)$ (in our example, Figure 3). In this case, we store the SubID in Tnode $_{001}$ and Tnode 0100 .

Given the Tnodes labels, we showed above that we can identify the sub-range for which the node is responsible for. However, the inverse operation (i.e., given the 
range find the node label, $(b l)$, is done with the following method. Based on the way sub-ranges are mapped to Tnodes, we can notice that for a given decomposed subrange $r$, the Tnode responsible for that subrange lays in depth $d=D-\log _{\beta}|r|-1$. Since we know the Tnode $l b l$ 's depth, its label is the $d+1$ prefix of the string representation in the $\beta-$ base alphabet of the lower bound of the subrange. That is: $l b l=\operatorname{prefix}(d+1, \beta-\operatorname{ary}(B l))$, where $\beta-\operatorname{ary}($ int $x)$ is the string representation of integer $x$ and $B l$ is the lower bound of the subrange. For example, as you can see in Figure 3, the decomposed subrange [3,4) is mapped to a Tnode in depth $4-0-1=3$, and since the binary representation of the lower bound, 3 in $D=4$ characters long string is '0011', Tnode's label is the $4(3+1)$ character long prefix of string '0011', resulting in '0011'.

When an event arrives declaring a value, we first compute the smallest possible subrange that includes the value. If, for example, the value is 3 , the smallest possible subrange including 3 , is $[3,4)$. Then, we compute the label of the Tnode responsible for that range, '0011', and route the event towards Tnode 0011 . All stored SubIDs from root to leaf Tnode $_{0011}$ are considered to match the event.

Given that we know in advance every attribute's domain bounds (low and high bounds, $B L, B H$ ) we can easily transform the predicates $\leq, \geq$, and $\neq$ into range predicates. For example, the predicate $\leq V$ can be thought as the range $[B L, V]$, the predicate $\geq V$ as $[V, B H]$ and the predicate $\neq V$ as $[B L, V)$ and $(V, B H]$.

\subsection{Load balancing issues}

A possible limitation of the approach already described is that a very small fraction of the nodes may become bottlenecks as they are expected to absorb the access load of incoming subscriptions and events. Nodes belonging to this category are all Tnodes close to the root of each one of the $T_{i}$ trees. In PastryStrings we adopt two widely used and complementary techniques for distributing the load: (i) replicating the forest structure among the network nodes and (ii) partitioning the stored subscriptions for a popular value. Further, we can achieve even more load distribution by applying domain relocation.

\section{Replicating the Forest}

Replicating the forest results in balancing the access load (for storing subscriptions or locating them when events arrive) across the network. We define the replication factor $(R F)$, as the number of replicas for each one of the $\beta$ string trees. For this to be done we could use a hash function returning randomly $R F$ values for each specific input. Thus, during the $T_{i}$ root look-up phase (when an event or subscription is looking for the $T_{i}$ tree) we could use this special function so as to reach one of the $R F$ different replica roots (and eventually trees) and then follow a path inside that replica tree.

During the subscription storing phase, we could either repeat the same $S u b I D$ storing process $R F$ times (for each replica tree) or let the node which was chosen to hold the subscription to inform the corresponding replica nodes for storing the subscription. Both approaches are easy to implement and details are omitted for space reasons.

\section{Partitioning the Storage Load}

In real applications it is likely that some Tnodes may become overloaded because of storing subscription ids defining a popular value. This kind of storage load hot spots may be avoided by defining a threshold for the number of stored SubIDs which when it is exceeded the Tnode chooses randomly another Tnode for further storing SubIDs . Each Tnode under this scheme maintains pointers to other Tnodes holding SubIDs for the same value, so as to be possible to collect all matched subscriptions for an incoming event.

\section{Numerical Attribute's Domain Relocation}

A typical PastryStrings configuration can support extremely large domains of integer values, $D m$. However, each numerical-typed attribute, $a_{i}$, is expected to have a much smaller domain, $D m_{i}$. Given that some ranges are expected to be very popular, a small set of Tnodes are expected to absorb a great load of requests for storing and retrieving SubIDs. With this observation in mind, we propose to distribute each $D m_{i}$ in our domain $D m$ adding an attributespecific base value, $b_{i}$, to each attribute's $i$ value. This kind of relocation will result in spreading the attributes' values across a large number of string trees and Tnodes and will then ameliorate load balancing problems.

\subsection{Self-organisation}

The self organisation of the string tree forest is required in highly dynamic $\mathrm{p} 2 \mathrm{p}$ networks with frequent node arrivals/departures and failure/recoveries. In order to treat failures successfully we must ensure string tree connectivity, so we need extra routing state per Tnode. This extra state, consists of pointers to a descendant node (that is a child of a Tnode's child) and two pointers to the left and right siblings in the $T_{i}$ tree structure. Due to space limitation we do not address here the proposed algorithms. However, interested readers may find more details in [3]. To address information (subscription) persistence issues, the replication technique outlined earlier can be used.

\section{Multi-dimensional events and subscrip- tions}

So far, we have presented PastryStrings under a singleattribute event/subscription schema. In real world pub/sub systems events and subscriptions are defined over a schema 
that supports $A$ attributes. Each attribute $a_{i}$ consists of a name, type, and a value $v\left(a_{i}\right)$. A $k$-attribute $(k \leq A)$ event is defined to be a set of $k$ values, one for each attribute. Similarly, a subscription is defined through an appropriate set of predicates over a subset of the $A$ attributes of the schema.

The allowed operators are: (i) prefix (e.g. $a b c^{*}$ ), (ii) suffix (e.g. $* a b c$ ), (iii) equality, and (iv) numerical range. An event matches a subscription if and only if all the attribute predicates of the subscription are satisfied.

The subscription identifier in our approach, $S u b I D$ is the concatenation of three parts: $c_{1}, c_{2}$, and $c_{3}$. $c_{1}$ represents the id of the node where the subscription arrived from a connected to that node client and keeps metadata information about the subscription, $c_{2}$ refers to the key of the subscription for identifying it among the stored ones at $c_{1}$, and $c_{3}$ is the number of declared attributes in the subscription.

\subsection{Processing incoming subscriptions}

We maintain four lists (initially empty) in every Tnode for every attribute $a_{i}$ of our schema. These are the $L_{a i-p r e f}$ and $L_{a i-s u f f}$ lists, where we store the SubIDs of the subscriptions that contain prefix or suffix predicates on attribute $a_{i}$, respectively, the $L_{a i-e q}$ list dedicated to equality predicates, and the $L_{a i-n u m}$ dedicated to numerical predicates.

Storing subscriptions is done by appropriately storing the $S u b I D$ in at least $c_{3}$ nodes $^{3}$ using the methodology presented earlier. Briefly, we process each attribute $a_{i}$ of the subscription and (i) when dealing with prefix predicate we store $S u b I D$ at $L_{a i-p r e f}$ of $\operatorname{Tnode}_{v\left(a_{i}\right)}\left(v\left(a_{i}\right)\right.$ is the attribute's value), (ii) when dealing with suffix predicate we invert $v\left(a_{i}\right)$ and store $S u b I D$ at $L_{a i-s u f f}$ of Tnode $\operatorname{inv}_{\left(v\left(a_{i}\right)\right)}$, (iii) when dealing with equality predicate we store $S u b I D$ at $L_{a-e q}$ of $\operatorname{Tnode}_{v\left(a_{i}\right)}$, and finally (iv) when dealing with numerical values we decompose the range into subranges and following the methodology presented earlier we store $S u b I D$ at the $L_{a i-n u m}$ of all appropriate Tnodes. Details are omitted for space reasons and can be found in [3].

\subsection{Event processing and matching}

Suppose now, that an event arrives at the system with $N_{a-\text { event }}$ attributes defined. The SubID Lists Collection Phase starts by processing each attribute separately. It first locates the root Tnode of the appropriate tree and then the

\footnotetext{
${ }^{3}$ If all attributes in the subscription involve predicates on strings then $c_{3}$ Tnodes must be reached. However, if ranges are defined, then each range $R$, may be translated into $O\left(\log _{\beta}(|R|)\right)$ string values and thus the number increases.
}

event is forwarded towards the Tnode $e_{v\left(a_{i}\right)}$. In each Tnode in the path towards Tnode ${ }_{v\left(a_{i}\right)}$, we collect all stored lists for the given attribute and send them to the next Tnode in the path. At each step of this process, we merge the previously collected lists of each kind resulting in four major lists which are finally returned back to the node where the event arrived where the matching is performed. Those lists are the $L_{a i-N U M E R I C A L}, L_{a i-E Q U A L I T Y}, L_{a i-P R E F I X}$, and $L_{a i-S U F F I X}$ lists $^{4}$.

The next step, termed Matching Phase, is actually the event-subscriptions matching process. Suppose, now, that a subscription $S u b I D_{k}$ is found to be in at least one of the collected lists. Assume that this subscription consists of $N_{a-s u b-k}$ attributes $\left(N_{a-s u b-k}\right.$ is obtained from the field $c_{3}$ of the subscription identifier). Then, this subscription is considered to match the event if it appears in exactly $N_{a-s u b-k}$ lists collected from the network, since an event matches a subscription if and only if all of the subscription's predicates are satisfied. Those $S u b I D s$ are then transferred to the Matching list $L_{\text {matching }}$ where they are processed further in order to inform the subscribers that are interested for the incoming event utilising field $c_{1}$.

A number of distributed algorithms for event matching can be used to avoid performance problems stemming from the use of a per-event coordinator for event matching. These are orthogonal issues and outside the scope of this paper. We refer the interested reader to [2].

\subsection{Message complexity analysis}

The Pastry infrastructure ensures that at most $O\left(\log _{\beta}(N)\right)$ messages are needed to reach any node in a system with namespace size $N$ and node identifiers of base $\beta$.

During the subscription storage procedure, the average number of messages needed to store a $S u b I D$ is equal for all allowable operations on strings. Thus, for stringtyped attributes we need $O\left(\log _{\beta}(N)\right)$ messages in order to reach the root of the appropriate $T_{i}$ string tree (i.e. one DHT lookup) and then at most $O\left(\log _{\beta}(N)\right)$ messages in order to locate the Tnode inside the string tree that will accommodate the subscription id (i.e. one message per string character), yielding a total of $O\left(\log _{\beta}(N)\right)$ messages. For numerical-typed attributes, if the mean size of ranges is $|R|$, the range decomposition and string translations used in RST results in $O\left(\log _{\beta}|R|\right)$ string values. Thus $O\left(\log _{\beta}|R| \times \log _{\beta}(N)\right)$ messages are required for storing a numerical range. $O\left(\log _{\beta}|R|\right)$ is expected to be small compared to $O\left(\log _{\beta}(N)\right)$ in real-life pub/sub applications with range sizes $(|R|)$ very small compared to $N$. Thus, we could view $O\left(\log _{\beta}|R|\right)$ as a relatively small constant.

\footnotetext{
${ }^{4}$ In fact in order to collect the $L_{a-S U F F I X}$ list we should repeat the same procedure with the inverted string value of the event.
} 
Regarding the matching process and more precisely the SubID Lists Collection Phase, for every attribute in the event, we should first locate the appropriate string tree, which requires $O\left(\log _{\beta}(N)\right)$ messages (i.e. one DHT lookup). Then we locate the right Tnode and then collect the SubID lists stored in all Tnodes in the path from the root to Tnode. This step requires at most $O\left(\log _{\beta}(N)\right)$ messages. Thus, in general, for each attribute of the event, $O\left(\log _{\beta}(N)\right)$ messages are required in order to collect the stored SubID lists.

\section{Experimentation and performance evalua- tion}

We performed a number of experiments in a 1000broker PastryStrings simulated network with up to 140,000 SubIDs stored and 160,000 generated requests for collecting $S u b I D$ lists (the exact number depends on the skewness of relevant distributions). We used a Zipfian ${ }^{5}$ popularity distribution for attributes, which determines the actual number of attributes in an event or subscription, varying from 1 to 10 . The popularity of values for each attribute also follows a Zipfian distribution. As the skewness of the values' distribution plays a key role here, we varied $\vartheta$ from 0.0 to 1.6 (to test for load imbalances). Regarding the distribution of numerical and string typed attributes in the subscriptions, half of the attributes are numerical (and the rest strings). Unless stated otherwise, half of the numerical attributes are declaring equalities while the other half ranges on integer numbers. The domain of each numerical attribute is $[0,70000]$ and the size of each range defined in subscriptions, unless stated otherwise, was varied from 1 (equality) to 20 (following a uniform distribution). The $\beta$ base of our alphabet was set to 15 and the maximum string length to 5 .

\subsection{The effect of ranges on subscription processing performance}

In the first set of experiments (detailed results are omitted for space reasons) we varied the percentage of attributes defining a numerical value (equality or range) in each subscription from $0 \%$ (no ranges at all) to $100 \%$. Our performance metric here is the number of messages needed to store a subscription. We observed that as the percentage of range predicates per subscription increases, so does the number of messages for subscription processing. In fact the message count for the only-ranges case was four times larger than the no-ranges case. This is as expected from our analysis since numerical attributes need to be decomposed

\footnotetext{
${ }^{5}$ the frequency of occurrence of the $n^{t h}$ ranked item is defined to be $\frac{1}{n \vartheta}$. Typical values of the parameter $\vartheta$ are: $0.0 \leq \vartheta \leq 1.6$, where large values of $\vartheta$ denote very skewed distributions and $\vartheta=0.0$ yields a uniform distribution
}

into subranges for each of which a different Tnode is responsible.

\section{$5.2 \quad$ Load balancing}

Our main objective with this set of experiments, is to observe how load balancing is affected by changing the skewness of the attributes' and values' popularity distribution.

\subsubsection{The data distribution effect on access and storage load}

Our specific performance metric here is the coefficient of variation $(\mathrm{CV})$ of access and storage load. $\mathrm{CV}$ for storage load is defined as the ratio of the standard deviation of the number of stored SubIDs in a network node across PastryStrings, to the mean value of stored SubIDs in a node. $\mathrm{CV}$ for access load involves both the number of requests for retrieving $S u b I D s$ and routing requests to nodes. As you can see in Figure 4(a) the coefficient of variation of storage and access load slightly increases as the attributes' skewness increases because the number of attributes per event/subscription decreases (fewer popular attributes are defined per event/subscription). Having fewer attributes results in more workload for fewer nodes. However, the difference between skewed and uniform workloads seems to be small.

Figure 4(b) shows how access and storage CV increases as values become more popular. This can be explained by the fact that as $\vartheta$ increases fewer and popular values are defined in each subscription/event. This results in overloading a few Tnodes in the PastryStrings infrastructure while others remain lightly-loaded. To study how storage load balancing can be improved by applying the attributes' domain relocation (recall the discussion in section 3.4) we made a number of experiments varying the attributes' values popularity distribution skewness and observed that the CV for storage is not affected by more skewed values' popularity distributions. This comes from the fact that range processing dominates string processing during the subscription storage phase (since ranges involve the contacting of many more Tnodes) and the domain relocation further distributes hot values and ranges to different Tnodes. Detailed results are omitted for space reasons.

\subsubsection{Effect of replication on access and storage load}

In this set of experiments we tried to measure how the replication of the string tree forest helps distributing evenly the storage and the access load during the SubID storage phase and the SubID collection phase, respectively, by varying the replication factor RF (the number of replica string tree forests). 

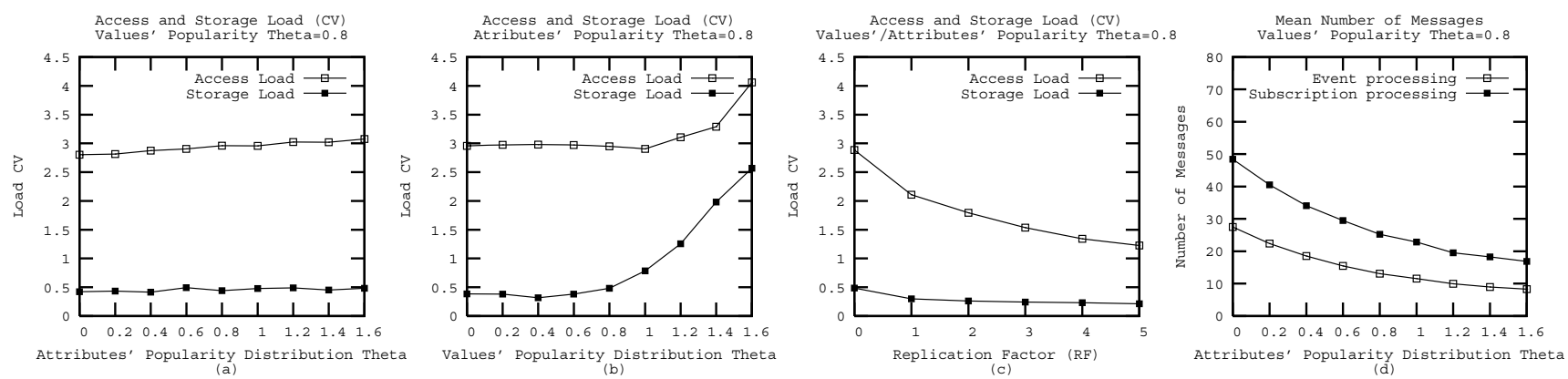

Figure 4. Access/Storage load balancing and messages needed for event/subscription processing.

As you can see in Figure 4(c) the coefficient of variation $(\mathrm{CV})$ of the number of SubIDs stored in network nodes (storage load) as well as the number of retrieval and routing requests for $S u b I D s$ (access load) decreases and approaches 0 (fully balanced), as the replication factor increases. Another important observation is that the network is more imbalanced when dealing with access load compared to the storage load. This is due to the fact that subscriptions involving range predicates may generate a greater number of storage requests (recall that the range decomposition may result in storing the $S u b I D$ in $\log _{\beta}|R|$ nodes). This number of SubIDs stored is greater and have a more evenly distribution among brokers, compared to the distribution of event requests.

\subsection{Event processing and matching}

\subsubsection{Number of messages}

We also conducted a number of experiments in order to measure the number of messages per event needed to collect all $S u b I D$ lists and the number of messages per subscription in order to store SubIDs as a function of the attributes' and values' popularity distribution skewness.

We observed that as the the skewness of the attributes' popularity distribution increases, fewer attributes are involved per event/subscription and thus the mean number of messages per event/subscription decreases (Figure 4(d)). Again, our main observation here is that subscription processing needs more communication overhead, compared to event processing. This is the right design choice. In real pub/sub systems events are expected to arrive in the system in rates much greater than subscriptions rates. Thus, it is deemed necessary to perform a fast and efficient event matching. We also increased the skewness of the values' popularity distribution and we have noticed that the number of messages per event/subscriptions is not affected by the value popularity.

\subsubsection{Network traffic}

Our specific performance metric here is the total number of SubIDs sent for the processing of each incoming event.

We varied the skewness of the attribute values' distribution while $\vartheta$ for the attributes' popularity equals 0.8 . Detailed results are omitted for space reasons. Briefly, we observed that as the values' popularity distribution becomes more skewed (varying $\vartheta$ of Zipfian popularity distribution from 0.0 to 1.6) the traffic increases by a factor of 5 since most of the incoming events, contact a small number of nodes that hold the majority of stored subscription identifiers. We also varied the attributes' popularity distribution (varying $\vartheta$ from 0.0 to 1.6 ) and we observed that when the distribution is skewed ( $\vartheta$ approaches 1.6) the network traffic is decreased by a factor of 2.5 , since fewer and popular attributes are chosen in every incoming event.

\section{Conclusions}

In this work we have contributed an architecture for building scalable, self-organising, well-performing systems that support queries with a rich set of predicates on string and numerical typed attributes. We specifically focused on and presented how our algorithms can be applied in a pub/sub environment with a broker network implemented using a DHT network. The distinguishing feature of our work is that is shows how to leverage specific DHT infrastructures to ensure logarithmic message complexity for both event and subscription processing, and for both rich string and numerical predicates. PastryStrings is DHT-specific, but does not interfere with the DHT internals; it simply leverages its key information.

Our experimentation results show that PastryStrings can handle subscriptions with rich string and numerical predicates efficiently and scalably, i.e., with small number of messages, good load distribution to network nodes, and small network bandwidth requirements. 


\section{Acknowledgements}

This work has been partially supported by the IST Programme of the European Union number IST-2004-001907 (DELIS) and the European Social Fund (ESF), Operational Program for Educational and Vocational Training II (EPEAEK II), and particularly the Program PYTHAGORAS.

\section{References}

[1] K. Aberer, $P$-grid: A self-organizing access structure for $p 2 p$ information systems, CoopIS'01, 2001.

[2] I. Aekaterinidis and P. Triantafillou, Internet scale string attribute publish/subscribe data networks, 14th ACM Conference on Information and Knowledge Management (CIKM05), 2005.

[3] I. Aekaterinidis and P. Triantafillou, PastryStrings: Contentbased publish/subscribe over dht networks, Tech. Report TR2006/04/01, R\&A Computer Technology Institute, Patras, Greece, 2006. (http://netcins.ceid.upatras.gr)

[4] G. Banavar, T. Chandra, B. Mukherjee, and J. Nagarajarao, An efficient multicast protocol for content-based publishsubscribe systems, 19th ICDCS, 1999.

[5] A. Carzaniga and A. Wolf, Forwarding in a content-based network, Proc. SIGCOMM'03, 2003, 2003.

[6] M. Castro, P. Druschel, A. Kermarrec, and A. Rowstron, Scribe: A large-scale and decentralized application-level multicast infrastructure, Journal on Selected Areas in Communication (2002).

[7] A. Crespo and H. Garcia-Molina, Routing indices for peerto-peer systems, ICDCS'02, 2002.

[8] G. Cugola, E. D. Nitto, and A. Fuggetta, The jedi event-based infrastructure and its application to the development of the opss wfms, (2001).

[9] P. Th. Eugster, P. Felber, R. Guerraoui, and A. M. Kermarrec, The many faces of publish/subscribe, ACM Computing Surveys, 2003.

[10] Jun Gao and Peter Steenkiste, An adaptive protocol for efficient support of range queries in dht-based systems, 12th IEEE International Conference on Network Protocols (ICNP'04), 2004.

[11] A. Gupta, O. D. Sahin, D. Agrawal, and A. El Abbadi, Meghdoot: Content-based publish subscribe over p2p networks, Middleware'04, 2004.

[12] M. Harren, J. M. Hellerstein, R. Huebsch, B. Thau Loo, S. Shenker, and I. Stoica, Complex queries in dht-based peerto-peer networks, IPTPS'02, 2002.

[13] R. Huebsch, J. M. Hellerstein, N. Lanham, B. Thau Loo, S. Shenker, and I. Stoica, Querying the internet with pier, VLDB'03, 2003.

[14] H. V. Jagadish and B. C. Ooi and Q. H. Vu, BATON: A Balanced Tree Structure for Peer-to-Peer Networks, VLDB'05,2005.
[15] S. W. Ng, B. C. Ooi, K. L. Tan, and A. Zhou, Peerdb: A p2pbased system for distributed data sharing, ICDE'03, 2003.

[16] P. R. Pietzuch and J. Bacon, Peer-to-peer overlay broker networks in an event-based middleware, DEBS03, 2003.

[17] C. G. Plaxton, R. Rajaraman, and A. W. Richa, Accessing nearby copies of replicated objects in a distributed environment, ACM Symposium on Parallel Algorithms and Architectures, 1997.

[18] S. Ramabadran, S. Ratnasamy, J. M. Hellerstein, and S. Shenker, Brief announcement: Prefix hash tree, ACM PODC, 2004.

[19] S. Ratnasamy, P. Francis, M. Handley, R. Karp, and S. Shenker, A scalable content addressable network, ACM SIGCOMM'01, 2001.

[20] Sean Rhea, Dennis Geels, Timothy Roscoe, and John Kubiatowicz, Handling churn in a dht, Proceedings of the 2004 USENIX Annual Technical Conference (USENIX '04), 2004.

[21] A. Rowstron and P. Druschel, Pastry: Scalable and distributed object location and routing for large-scale peer-topeer systems, ACM Middleware’01, 2001.

[22] I. Stoica, R. Morris, D. Karger, F. Kaashoek, and H. Balakrishnan, Chord: A scalable peer-to-peer lookup service for internet applications, SIGCOMM'01, 2001.

[23] C. Tang and Z. Xu, pFilter: Global Information Filtering and Dissemination, IEEE FTDCS'03,2003.

[24] W. W. Terpstra, S. Behnel, L. Fiege, A. Zeidler, and A. P. Buchmann, A peer-to-peer approach to content-based publish/subscribe, DEBS'03, 2003.

[25] P. Triantafillou and I. Aekaterinidis, Publish-subscribe over structured $p 2 p$ networks, DEBS 04, 2004.

[26] P. Triantafillou and A. Economides, Subscription summarization: A new paradigm for efficient publish/subscribe systems, IEEE ICDCS04, 2004.

[27] P. Triantafillou and A. Economides, Subscription summaries for scalability and efficiency in publish/subscribe systems, DEBS'02, 2002.

[28] C. Tryfonopoulos, S. Idreos, and M. Koubarakis, Publish/subscribe functionality in ir environments using structured overlay networks, In Proc. of ACM SIGIR, 2005.

[29] A. L. Wolf, A. Carzaniga, and D. S. Rosenblum, Achieving scalability and expressiveness in an internet-scale event notification service, ACM PODC, 2000.

[30] B. Yang and H. Garcia-Molina, Improving search in peer-topeer systems, ICDCS'02, 2002.

[31] B. Y. Zhao, L. Huang, J. Stribling, S. C. Rhea, A. D. Joseph, and J. D. Kubiatowicz, Tapestry: A global-scale overlay for rapid service deployment, IEEE Journal on Selected Areas in Communications (2003).

[32] D. Tam and R. Azimi and H-A. Jacobsen, Building ContentBased Publish/Subscribe Systems with Distributed Hash Tables, Proc. DBISP2P'03, 2003. 